\section{FDA approves ultra-orphan drug on a 4-patient trial}

US regulators have approved Wellstat Therapeutics' uridine triacetate for the treatment of hereditary orotic aciduria (HOA), an ultra-orphan indication that has been reported in only 20 people worldwide.

$\mathrm{HOA}$ is an inherited disease that is caused by a defect in the gene that encodes uridine 5'-monophosphate synthase. This leads to an inability to normally synthesize uridine, a necessary component of RNA, and causes haematologic abnormalities including anaemia, leukopenia and neutropenia. In case studies of the disease, oral administration of uridine improved haematologic abnormalities in patients.

Uridine triacetate is an acylated form of uridine that is absorbed into the blood better than is uridine itself. Given the tiny HOA patient population, the US Food and Drug Administration approved the drug on the basis of results from a 4-patient 6-week clinical trial with a 6-month extension phase. Treatment improved the stability of haematologic parameters in all four patients. No side effects were observed with treatment.

Wellstat has not yet disclosed pricing for the ultra-orphan drug.

Wellstat also received a priority review voucher - which allows a sponsor to get a drug reviewed in 8 months rather than the standard 12 months - because the drug was approved for a rare paediatric indication. The company sold the voucher to AstraZeneca for an undisclosed sum, making it the fifth priority review voucher to be sold. Previous vouchers have sold for US \$67.5 million-\$350 million. The July approval of Sanofi and Regeneron's proprotein convertase subtilisin-kexin type 9 (PCSK9)-specific antibody alirocumab marked the first successful use of a priority review voucher.

Asher Mullard

\section{FDA approves female sexual dysfunction drug}

US regulators have approved Sprout Pharmaceuticals' flibanserin for hypoactive sexual desire disorder in premenopausal women. Regulators previously rejected the controversial drug twice, and the change in decision prompted critics to raise concern that the approval was a triumph of marketing over science.

Flibanserin is a serotonin receptor $1 \mathrm{~A}$ agonist and a serotonin receptor $2 \mathrm{~A}$ antagonist that was initially developed as an antidepressant. Its mechanism of action in hypoactive sexual desire disorder is unknown. In three randomized trials of the drug in a total of 2,400 premenopausal women, the drug increased the number of satisfying sexual events by $0.5-1$ events per month over placebo. It also increased sexual desire on average by $0.3-0.4$ points (on a 3-point scale) over placebo. Adverse events included low blood pressure and loss of consciousness, especially in women who drank alcohol.

The drug, developed and first filed by Boehringer Ingelheim, was initially rejected on the basis of results from two pivotal trials in 2010. At that time, a US Food and Drug Administration (FDA) advisory panel voted unanimously that the drug's side effects were unacceptable and voted 10 to 1 that the data did not demonstrate efficacy. Boehringer discontinued development of the drug after the rejection, and sold it to Sprout Pharmaceuticals in 2011. Sprout resubmitted the drug in 2013, with data from a third pivotal trial, only to have it rejected again that year.

Sprout resubmitted the drug this year with additional safety data. At an FDA advisory meeting in June, independent experts voted 18 to 6 to approve the drug with a risk evaluation and mitigation strategy (REMS). This vote and the approval a few months later led some critics to note that marketing tactics - including an effort by 'Even The Score', a Sprout-funded advocacy group, to portray the previous rejections as a 'gender equality issue' at the FDA — swayed the decision.

"The Even the Score advocacy campaign, the shifting efficacy end points and use of a patient-reported outcome measure, the tenuous risk-benefit balance among the studied population and potential for widespread off-label use, and an unmet medical need $[. .$.$] are not totally unfamiliar territory$ for the FDA, but represent a challenge when FDA's independent advisors after the approval (IAMA 314, 869-870; 2015). "What makes the approval process for flibanserin even more unique is the politically charged atmosphere in which the FDA will decide how all these trade-offs should best be navigated."

Days after the approval, Valeant bought Sprout for US\$1 billion.

Asher Mullard they occur simultaneously," wrote three of the

\section{Off-label targeted cancer drugs fail in first randomized trial}

Encouraged by a growing arsenal of targeted cancer drugs and the ability to molecularly profile tumours, oncologists are increasingly using targeted drugs off-label to treat patients. Up to $30 \%$ of cancer drug use is off-label. A first randomized trial of off-label targeted drugs versus chemotherapy has now shown that this personalized approach does not benefit patients (Lancet Oncol. 2 Sep 2015 [epub ahead of print]).

Christophe Le Tourneau, an oncologist at the Institut Curie in Paris, France, and the lead author of the study, randomized 195 patients with 'actionable' abnormalities in a range of cancers to one of two arms: treatment with a potentially relevant, targeted drug, or treatment with chemotherapy. Progression-free survival was 2.3 months in the experimental personalized-treatment arm, non-significantly different from the 2.0 months on chemotherapy. There was a non-significant trend towards increased toxicity with the targeted drugs.

"Our trial has drawbacks," admits Le Tourneau. It was a small trial, in patients with advanced cancer who may have been unlikely to benefit. The investigators were also limited in terms of the drugs that they could use (only approved drugs), and applied a simplistic algorithm for matching molecular abnormalities to targeted drugs. But many of these limitations also apply for oncologists who use drugs off-label in the clinic, he adds.

The trial "offers robust evidence for the deficiencies in assigning therapy based on the various loose associations between biomarkers and inhibitors that are often provided in commercial clinical diagnostic reports. The results suggest that off-label use of molecularly targeted agents in this manner should be restricted," writes Daniel Catenacci, an oncologist at the University of Chicago, Illinois, USA, in an editorial about the trial (Lancet Oncol. 2 Sep 2015 [epub ahead of print]).

Several other trials are also ongoing to test the broader use of targeted cancer drugs, including the $\mathrm{NCl}-\mathrm{MATCH}$ trial, which will test 25 targeted drugs in 1,000 patients with different tumour types (Nat. Rev. Drug Discov. 14, 513-515; 2015).

Asher Mullard 